\title{
The importance of individualized pharmaceutical therapy in the treatment of diabetes mellitus
}

\author{
David B. Nash \\ Thomas Jefferson University \\ Jennifer B. Koenig \\ Thomas Jefferson University \\ Karen D. Novielli \\ Thomas Jefferson University \\ Renee Liberoni \\ Thomas Jefferson University Hospital \\ Miriam Reisman \\ Thomas Jefferson University \\ Follow this and additional works at: https://jdc.jefferson.edu/healthpolicyfaculty \\ Part of the Health Services Research Commons \\ Let us know how access to this document benefits you
}

\section{Recommended Citation}

Nash, David B.; Koenig, Jennifer B.; Novielli, Karen D. ; Liberoni, Renee; and Reisman, Miriam, "The importance of individualized pharmaceutical therapy in the treatment of diabetes mellitus" (2001). College of Population Health Faculty Papers. Paper 21.

https://jdc.jefferson.edu/healthpolicyfaculty/21

This Article is brought to you for free and open access by the Jefferson Digital Commons. The Jefferson Digital Commons is a service of Thomas Jefferson University's Center for Teaching and Learning (CTL). The Commons is a showcase for Jefferson books and journals, peer-reviewed scholarly publications, unique historical collections from the University archives, and teaching tools. The Jefferson Digital Commons allows researchers and interested readers anywhere in the world to learn about and keep up to date with Jefferson scholarship. This article has been accepted for inclusion in College of Population Health Faculty Papers by an authorized administrator of the Jefferson Digital Commons. For more information, please contact: JeffersonDigitalCommons@jefferson.edu. 


\title{
The Importance of Individualized Pharmaceutical Therapy in the Treatment of Diabetes Mellitus
}

\author{
DAVID B. NASH, M.D., M.B.A., JENNIFER B. KOENIG, M.S., KAREN D. NOVIELLI, M.D., \\ RENEE LIBERONI, M.P.H., and MIRIAM REISMAN, M.F.A.
}

\begin{abstract}
Individualized pharmaceutical care for patients with diabetes is necessary for several reasons. First, diabetes is a highly complex disease caused by the interplay among genetic, physiological, and environmental factors that vary from individual to individual. Second, the profile of patients with diabetes has evolved to include people of all ages and socioeconomic backgrounds, with varying medical histories and health behaviors. Third, diabetes often occurs concurrently with other medical conditions, especially in certain groups, such as the elderly. While the treatment goals for all patients with diabetes are the same-to stabilize and maintain healthy blood glucose levels to prevent serious complications-the treatment plan used to achieve those goals will vary among individuals. There are many clinically proven options available for the treatment of diabetes. While there are well-established guidelines regarding which intervention is the best option for patients with either type 1 or type 2 diabetes, individual patient characteristics and needs should drive the care process. Assuring patient access to the wide variety of medications is crucial to meeting these needs and achieving quality, cost-effective diabetes management.
\end{abstract}

\section{EXECUTIVE SUMMARY}

D IABETES MELLITUS is a complex disease with a prevalence that is rapidly growing, especially among certain groups of Americans. In the United States nearly 6\% of the total population (approximately 16 million people) has diabetes. ${ }^{1}$ Hispanics, African Americans, the elderly, children, and the clinically obese are at high risk for the disease. ${ }^{1-5}$ The impact of diabetes is felt economically, clinically, and socially. This chronic condition drains limited healthcare dollars, tests the boundaries of clinical resources, and decreases quality of life for patients and their families.

The insidious nature of diabetes compounds the threats it poses; up to one third of people with diabetes in America are undiagnosed, ${ }^{1}$ despite the fact that diabetes can be identified through a simple blood test. ${ }^{3}$ But patients are often asymptomatic in the early stages, going untreated until their bodies have sustained irreversible damage. These effects take a high toll on individual patients and on America's healthcare system overall. Diabetes is the sixth leading cause of death from disease, and its combined direct and indirect costs total nearly $\$ 100$ billion annually. $^{2}$

Controlling diabetes is a clinical challenge for several reasons. First, diabetes is a highly complex illness caused by the interplay among genetic, physiologic, and environmental factors 
that vary from individual to individual. ${ }^{6}$ Careful monitoring and intensive therapy are required to avoid serious complications. As environmental and physiologic factors fluctuate, drug therapy that was once effective may require modification.

Second, the diabetic population is changing. At one time, diabetes was primarily considered a disease of people with atypical genes (type 1) or long lifespans (type 2). Now, epidemiologists know that diabetes affects a larger, more diverse group of people. While socioeconomic and technological changes have caused Americans to live longer (thanks to modern medicine), they have also encouraged unhealthy lifestyles (thanks to modern conveniences), putting more people at greater risk for developing diabetes today. ${ }^{7-8}$ In addition, there is greater diversity among individuals who develop diabetes. For example, in the past, diabetes in children was almost always type 1; recently, increases in childhood type 2 diabetes have been reported. ${ }^{1}$ Although similar in age, a child with type 1 diabetes would need a different treatment plan than a child with type 2 diabetes. Greater patient diversity warrants greater therapeutic diversity. ${ }^{9}$

Third, diabetes often occurs along with other medical conditions, especially in the elderly. ${ }^{11-14}$ Diseases that frequently coexist with diabetes include cardiovascular disease, depression, obesity, and arthritis. Diabetes with comorbid conditions presents a greater treatment challenge due to potential disease synergies, drug interactions, and compromised physiologic reserves. ${ }^{9}$

The profile of patients with diabetes has evolved to include people of all ages and socioeconomic backgrounds, with varying medical histories and health behaviors. As the patient population diversifies, management of diabetes must address the differences among individual patients. ${ }^{9}$ While the treatment goals for all people with diabetes are the samelize and maintain healthy blood glucose levels to prevent serious complications-the treatment plan used to achieve those goals will vary. Imagine three patients: an 8-year-old male with type
1 diabetes but no other health problems, a 48year-old female with mild type 2 diabetes and coexisting heart disease, and a 72-year-old male with advanced type 2 diabetes and significant diabetes-related sequelae. The clinical differences alone call for somewhat different treatment plans. When socioeconomic variables are added to the mix (e.g., diet, exercise, living environment, availability of medications, involvement of caregivers), markedly different management strategies may be required.

Fortunately, the past few decades have seen the development of a myriad of treatment options to address the specific needs of individuals with diabetes. Breakthrough innovations include the synthesis of human insulin in the 1980s and effective insulin analogues with improved properties in the 1990s. Incremental innovations in pharmacotherapy have been equally valuable in children and elderly patients with diabetes. ${ }^{9}$ For instance, alternative dosage forms encourage patient adherence by meeting patient-specific needs, ${ }^{15}$ and newer insulin analogues offer improved side effect profiles. ${ }^{16}$ Recombinant DNA technology has made some of these innovations possible, and biomedical technologies will continue to play an important role in future research and development. ${ }^{17-19}$ Treatments for type 2 diabetes have also been expanded to include more than 20 different medications that reduce blood glucose levels in several different ways. The variety of medications helps patients who are prone to side effects or have difficulty adhering to their regimens to find a management plan that is effective for them. Newer oral diabetes medications have reduced the potential for hypoglycemic reactions, and increased options encourage adherence to treatment plans.

Yet despite the improvements in diabetes treatment and education, the prevalence of the terrible consequences of diabetes continues to increase at an alarming rate. Therefore, still greater efforts are necessary. To this end, we offer this paper as a diabetes management approach that may help thwart the disease and its high human and economic costs. This approach focuses on the benefits of individual- 
ized diabetes care. With the many pharmaceutical options available today, treatment can be tailored to specific patients and groups. But the availability of these medications is not enough. High-quality, cost-effective diabetes care can be achieved only if: (1) the patient's specific needs are accurately identified, and (2) there is access to the wide variety of diabetes drugs to match these needs. To foster a successful match between each patient and his or her drug therapy, many factors-clinical, social, and economic-must be considered. The final section of the paper provides a simple tool that encourages patients, providers, and policy-makers to bear in mind the key aspects of individualized care in the fight against diabetes.

\section{INTRODUCTION: DIABETES BASICS}

\section{Epidemiology of diabetes}

In the United States nearly $6 \%$ of the total population (or 15.7 million people) has diabetes. Diabetes contributes to more than 193,000 deaths per year, and its annual incidence has increased sixfold since $1958 .{ }^{1}$ Diabetes has become more prevalent over the last two decades. The incidence of diabetes began rising in the early 1980s, leveled off in the middle of the decade, and then increased sharply in the 1990s. Between 1980 and 1996, the number of persons with diagnosed diabetes in the United States increased from 5.8 million to 8.5 million. ${ }^{20}$ By 1997, the number of persons with diagnosed diabetes had risen to 10.3 million, with 798,000 new cases diagnosed per year; an additional 5.4 million are believed to have undiagnosed diabetes. Diabetes is a growing problem among the elderly, children and adolescents, and the clinically obese. Certain ethnic groups, such as Native Americans, Latinos, and African Americans, are also at greater risk for the disease (Table 1). ${ }^{2,3}$

The number of persons with diagnosed diabetes, particularly type 2 diabetes, is increasing for several reasons: (1) improvements in screening and change in the diagnostic criteria from a fasting plasma glucose greater than or equal to 140 to one that is greater than or equal
Table 1. Prevalence of Diabetes in Different Ethnic Groups Compared to Overall U.S. Population

Percentage of persons

Subgroup with diabetes

Puerto Ricans 26

Mexican Americans

24

Cuban Americans

16

Native Americans

African Americans

12

Overall U.S. population

to 126 ; (2) increasing rates of obesity and sedentary lifestyles; (3) growth in populations that have high rates of type 2 diabetes $^{21}$; (4) recognition that type 2 diabetes is an increasing problem in children and adolescents ${ }^{22}$; and (5) an aging population.

\section{Definition of types 1 and 2}

Diabetes mellitus is a group of diseases in which hyperglycemia (elevated blood glucose) results from inadequate production or use of insulin, the hormone secreted by the pancreas that lowers blood sugar. Insulin is an essential element for many bodily functions: it allows glucose (sugar) to enter body cells where it is used as energy, and it helps the body synthesize protein and store fat. When insulin availability or function is compromised, glucose and lipids remain in the bloodstream instead of being converted to energy. Over time, excess glucose and lipids damage the body's organs, having particularly deleterious effects on the heart. ${ }^{1}$

Diabetes occurs in two forms: type 1, previously called insulin-dependent diabetes mellitus, and type 2, previously called non-insulin-dependent diabetes mellitus. Table 2 compares the features of the two major types of diabetes. In type 1 diabetes, the body completely lacks insulin. This type of diabetes generally appears in patients before the age of 18 and accounts for about $5 \%-10 \%$ of all cases of diabetes mellitus. It is caused by an autoimmune process that destroys the insulin-producing cells of the pancreas. Although a diabetes-specific diet, moderate exercise, and other healthy habits can help patients reduce 
Table 2. Comparison of Type 1 and Type 2 Diabetes

\begin{tabular}{|c|c|c|}
\hline & Type 1 diabetes & Type 2 diabetes \\
\hline Prevalence & $5-10 \%$ of cases & $90-95 \%$ of cases \\
\hline Age of onset & Usually before 18 & Usually after 45 \\
\hline Cause & $\begin{array}{l}\text { Body's inability to produce } \\
\text { insulin }\end{array}$ & $\begin{array}{l}\text { Body's inability to produce } \\
\text { enough insulin or resistance to } \\
\text { insulin }\end{array}$ \\
\hline Common symptoms & $\begin{array}{l}\text { Frequent urination or need to } \\
\text { urinate; excessive thirst; } \\
\text { extreme hunger; fatigue; } \\
\text { unusual weight loss; irritability }\end{array}$ & $\begin{array}{l}\text { Same as type } 1 \text { symptoms } \\
\text { and blurred vision; tingling in } \\
\text { hands or feet; increased } \\
\text { susceptibility to infection; slow } \\
\text { to heal cuts and bruises; } \\
\text { recurring skin, gum or bladder } \\
\text { infections }\end{array}$ \\
\hline $\begin{array}{l}\text { Number with disease who } \\
\text { develop symptoms }\end{array}$ & $100 \%$ & $33 \%$ \\
\hline Risk factors & $\begin{array}{l}\text { Exposure to environmental } \\
\text { triggers (i.e., unidentified } \\
\text { virus); having sibling or parent } \\
\text { with type } 1 \text { diabetes }\end{array}$ & $\begin{array}{l}\text { Obesity; advancing age (45+); } \\
\text { family history; ethnicity; } \\
\text { malnutrition; inactivity; high } \\
\text { cholesterol levels; history of } \\
\text { gestational diabetes; having a } \\
\text { baby weighing } 9 \text { lbs. or more at } \\
\text { birth }\end{array}$ \\
\hline Treatment & $\begin{array}{l}\text { Diet control; exercise; home } \\
\text { blood glucose testing; and } \\
\text { daily insulin injections }\end{array}$ & $\begin{array}{l}\text { Diet control; exercise; home } \\
\text { blood glucose testing; and, in } \\
\text { some cases, oral medication } \\
\text { and/or insulin }\end{array}$ \\
\hline
\end{tabular}

their glucose levels and avoid complications, type 1 diabetes always requires insulin therapy to counterbalance the absence of naturally occurring insulin. ${ }^{23}$

Type 2 diabetes is the most common form of diabetes, representing $90 \%-95 \%$ of all cases diagnosed. In type 2 diabetes, the body is either deficient in its insulin production, or resistant to insulin, or both. Insulin resistance occurs when the body's cells no longer respond properly to insulin and their ability to take up glucose is impaired, although insulin is available. Most type 2 diabetes develops in adulthood, although recent increases in children represent an alarming trend. Their increases appear to correspond with increases in obesity and inactivity among this age group. Generally, patients with type 2 diabetes are obese. ${ }^{23}$ Other rare, nonchronic forms of diabetes account for $1 \%-2 \%$ of all diagnosed cases.

\section{Symptomatology}

Although all people with type 1 diabetes develop symptoms, only approximately one third of type 2 sufferers do, perhaps accounting for the large number of undiagnosed cases. Symptoms common to both types of diabetes include frequent urination or need to urinate, excessive thirst, extreme hunger, fatigue, unusual weight loss, and irritability. ${ }^{24}$

\section{Risk factors and diagnostics}

Although diabetes goes undetected in many people, a lack of diagnostic tests is not the problem. Diagnosing diabetes is as simple as determining a plasma glucose level using a simple blood test. Two consecutive days of either a fasting glucose level of $126 \mathrm{mg} / \mathrm{dL}$ or greater or a nonfasting glucose level of $200 \mathrm{mg} / \mathrm{dL}$ or greater with symptoms indicates diabetes. ${ }^{3}$

The real problem with diagnosing diabetes is getting people at risk tested for the disease. Many people are not aware that they are at risk or already have diabetes. Risk factors for type 2 diabetes include obesity, advanced age (45 years and over), family history, ethnicity, malnutrition, inactivity, dyslipidemia (high choles- 
terol levels), and history of gestational diabetes or bearing a child weighing more than nine pounds at birth..$^{23}$ Anyone with one or more of these risk factors should be tested for diabetes.

\section{Consequences of untreated or inadequately treated diabetes}

Untreated type 1 diabetes has serious repercussions, specifically ketoacidosis. Ketoacidosis, also known as diabetic coma, is a metabolic crisis that occurs when poorly controlled diabetes causes high blood glucose levels. If no medical intervention occurs, ketoacidosis can lead to coma and death. When type 2 diabetes goes undetected and untreated, complications develop more slowly than in type 1 diabetes but can be just as serious. ${ }^{23,24}$ These complications may include one or more of the following conditions: eye disease and blindness, nerve damage, kidney failure, foot and leg ulcers that can lead to amputation, heart disease, stroke, and narrowing of the arteries. These complications also may occur in patients with type 1 disease.

Untreated or inadequately treated diabetes and its resultant complications cause substantial clinical and economic burdens of nearly $\$ 100$ billion annually. Substantial costs are incurred not only for diabetes-related direct medical expenditures, but also for the indirect costs of disability and lost productivity resulting from diabetes-related morbidity and premature mortality. ${ }^{25}$ According to the American Diabetes Association, the total estimated annual economic cost of diabetes in the United States in 1997 was $\$ 98$ billion dollars. This figure includes $\$ 44$ billion in direct medical and treatment costs and $\$ 54$ billion for indirect costs attributed to disability and mortality. In 1997, the per capita costs of healthcare for people with diabetes amounted to $\$ 10,071$, compared with $\$ 2,699$ for people without diabetes. ${ }^{25}$

\section{Diabetes management}

Studies have proven that diabetic complications can be minimized or avoided by maintaining normal or near-normal blood glucose levels. The Diabetes Control and Complica- tions Trial (DCCT), a landmark 10-year clinical study, established that intensive treatment of diabetes delays the onset and slows the progression of diabetes complications. ${ }^{27}$ The DCCT confirmed that maintaining a healthy blood glucose level at an average of $155 \mathrm{mg} / \mathrm{dL}$ reduces the long-term risk of nerve and eye damage and kidney disease. ${ }^{27}$ The patients studied in the DCCT were under 40 years old and were diagnosed with type 1 diabetes; however, analogous findings have been documented in older patient populations with either type 1 or type 2 diabetes.

Early intervention may be the key to limiting the severity of diabetes complications. ${ }^{28}$ The United Kingdom Prospective Diabetes Study (UKPDS), the most comprehensive study of patients with type 2 diabetes to date, followed 5,102 persons newly diagnosed with type 2 diabetes for an average of 10 years. This study confirmed and extended previous evidence in support of the hypotheses that hyperglycemia is a major cause of the microvascular complications of diabetes, and that cardiovascular outcomes are consistently associated with hyperglycemia irrespective of its underlying cause. ${ }^{29}$

Based on the DCCT and other important diabetes studies, the American Diabetes Association states that strict blood glucose control can have similar benefits in all types of diabetes because the complications of uncontrolled hyperglycemia develop through similar pathologies, regardless of how the underlying disease occurs. In other words, while type 1 and type 2 diabetes may not always be treated using the same therapies, the end result of any effective therapy - a consistently healthy blood glucose level-will prevent or delay complications in either condition. Details about diabetes treatment options are described in the subsequent section.

\section{PHARMACOLOGIC MANAGEMENT OPTIONS FOR DIABETES}

There are many clinically proven pharmacologic and nonpharmacologic interventions to 
control blood glucose levels for people with diabetes. Nonpharmacologic interventions include diet, exercise, and other behavioral modifications. Proper nutrition and exercise are a fundamental part of treating both type 1 and type 2 diabetes.

Pharmacologic treatment guidelines differ for type 1 and type 2 diabetes. Because people with type 1 diabetes cannot produce any insulin, they must inject it daily. Insulin reduces glucose levels by suppressing glucose production in the liver and by increasing glucose utilization after meals. ${ }^{30}$ A patient with type 2 diabetes may require oral drug therapy alone or in combination with insulin therapy.

Well-established clinical practice recommendations generally guide the decision regarding which intervention is the best option for diabetes patients. ${ }^{26}$ But individual patient characteristics should drive the care process, suggesting where interventions should begin, and in which direction care will proceed. Because diabetes is a progressive, incurable illness, the pathways are multidirectional and dynamic; that is, interventions almost always need modification over a patient's lifetime.

\section{Nonpharmacologic management: the first line of defense against diabetes}

The first interventions in diabetes management are nonpharmacologic. Even for patients with type 1 diabetes, for whom insulin therapy is always required, it is also important to use nonpharmacologic strategies to control blood glucose levels and to improve general health. For patients with type 2 diabetes, nonpharmacologic interventions may be enough to control diabetes, at least initially. Nonpharmacologic interventions control blood glucose levels by balancing insulin production and sensitivity with nutrition and exercise. Firstline therapy for type 2 diabetes calls for an adjustment in diet and exercise; for overweight patients, often a short trial of weight reduction and exercise is recommended before the initiation of pharmacotherapy. ${ }^{26}$ Exercise can facilitate weight loss, and it helps to improve insulin sensitivity, allowing the body to use insulin more efficiently. In addition to lowering blood glucose levels, diet and exercise can help improve a patient's lipid profile (i.e., reduce cholesterol levels), which is important for avoiding the cardiovascular complications of diabetes. Other behavior modifications, such as avoiding alcohol and tobacco, can also help manage diabetes and its complications.

\section{Oral drug therapy}

If and when nonpharmacologic treatment no longer controls blood glucose levels adequately, oral antidiabetic medications are generally the next step for people with type 2 diabetes. These medications may be used alone, in combination with one another, or as an adjunct to insulin. Oral medications for diabetes reduce blood glucose levels in several different ways. Some increase the amount of insulin secreted from the pancreatic beta cells. Others allow the body to use the insulin that is already present more effectively. A third group reduces the breakdown of carbohydrates in the gastrointestinal tract so that less sugar is absorbed. Some medications use a combination of these methods to manage blood glucose levels.

No single currently available agent appears to be superior when used as monotherapy, and combination therapy is often indicated. Many people with diabetes need to use more than one kind of medication to manage both their insulin production and insulin resistance. If a patient does not respond to a first-line agent, a second agent is typically added, not substituted. ${ }^{31}$

It is important to note that people with type 2 diabetes are not the only patients who may benefit from noninsulin oral medications. Although oral antidiabetic agents cannot be used as primary therapy for persons suffering from type 1 diabetes, these individuals may also benefit from therapy with a combination of oral drugs and insulin. For example, $\alpha$-glucosidase inhibitors used in addition to an insulin regimen have decreased the early peak in blood glucose following meals, thus minimizing the subsequent drop in plasma glucose, evening out glucose levels overall. ${ }^{32}$ 
The remainder of this section describes the major classes of noninsulin oral drug therapy available for the treatment of diabetes.

Sulfonylureas. Sulfonylureas work in two ways: they increase insulin production and reduce insulin resistance. They are taken one or more times a day and require careful monitoring to avoid a low blood sugar reaction (hypoglycemia). This class includes the following drugs:

First Generation

- Acetohexamide

- Chlorpropamide

- Tolazamide

- Tolbutamide

The decision to use a sulfonylurea and the choice among the available agents will depend greatly on the individual characteristics of the patient. Generally, second-generation sulfonylureas are preferable because they interact with fewer drugs and have a more favorable side effect profile. They are long-acting, allowing for once-daily dosing that may encourage adherence to drug regimens. Sulfonylureas with short half-lives tend to minimize hypoglycemic episodes; patients prone to such episodes will usually benefit from shorter acting agents such as glipizide and should avoid the long-acting medications such as chlorpropamide. Patients with compromised kidney or liver function should be monitored especially closely when taking sulfonylureas because these drugs are processed renally and hepatically. This applies to the elderly and patients with comorbid disease(s) that affect these organs. ${ }^{9}$ For these individuals, dosages should begin low (about half the recommended starting dose) and be titrated cautiously. Sulfonylureas are not indicated for patients with severe kidney or liver disease.

Biguanide. An alternative or adjunct to sulfonylureas is metformin, the only currently available biguanide. It controls blood glucose levels by acting on the liver to ensure that it does not produce excessive amounts of sugar. ${ }^{16}$ An additional benefit of metformin is that it promotes weight loss and reduction in blood fat and cholesterol levels, all of which can contrib- ute to better blood glucose control. However, alcohol use or kidney disease may preclude the use of this agent. Metformin (Bristol-Myers Squibb Co., Princeton, NJ) can also encourage anorexia, so it may not be preferable for older patients for whom malnutrition is a concern. In general, though, this agent is a good option for the elderly because unlike sulfonylureas and insulin, metformin does not contribute to hypoglycemia. ${ }^{9}$ Metformin is generally taken two or three times per day with meals.

$\alpha$-Glucosidase inhibitors. $\alpha$-Glucosidase inhibitors function by blocking gastrointestinal enzymes that digest carbohydrates, thus slowing and reducing the production of sugar from these foods. Two $\alpha$-glucosidase inhibitors are currently available: acarbose and miglitol. To maximize effectiveness, these medications are taken three times daily at mealtime. Like metformin, $\alpha$-glucosidase inhibitors do not cause hypoglycemia when taken alone. Acarbose also has a stool-softening effect that may be desirable in patients prone to constipation. ${ }^{16}$

Thiazoldinediones. The thiazolidinediones include rosiglitazone and pioglitazone. The first thiazolidinediones, troglitazone, was removed from the U.S. market because of rare but severe hepatotoxicity. ${ }^{16}$ Thiazolidinediones are indicated for type 2 diabetes; they sensitize the body to its own insulin or to insulin therapy. They can be used alone or with other medications such as metformin, sulfonylureas, or insulin; however, they have not been tested with $\alpha$-glucosidase inhibitors. Taken alone, thiazolidinediones do not cause hypoglycemia; with insulin or insulin analogues hypoglycemia can occur. The currently available thiazolidinediones can have a positive effect on the lipid profiles, and they do not have adverse effects on the liver or kidneys. Thiazolidinediones can reduce the effectiveness of oral contraceptives.

New combined oral medications. In August 2000, the Food and Drug Administration (FDA) approved the first fixed dose combination oral antidiabetic, Glucovance (Bristol-Myers Squibb 
Co., Princeton, NJ). Glucovance combines the two most often prescribed oral diabetes medications-glyburide and metformin-in one tablet. This new approach to the management of type 2 diabetes is indicated as first-line therapy for newly diagnosed diabetes, and is also indicated as second-line therapy for patients who have been taking metformin or a sulfonylurea alone without clinical success. ${ }^{34}$

Insulin and insulin analogues. Insulin or insulin analogs are required for all people with type 1 diabetes and some people with type 2 diabetes to maintain adequate blood glucose control. Because of progressive decline in beta cell function in type 2 diabetes, even the use of multiple oral medications is sometimes not adequate, and eventually many people with type 2 diabetes will require insulin therapy either in combination with the oral therapies or instead of them. ${ }^{30}$ Currently $40 \%$ of people with type 2 diabetes use insulin. The need for insulin increases with duration of the disease. ${ }^{30}$

Today, there are more than 20 types of FDAapproved insulin available in the United States. ${ }^{35}$ Insulin therapies vary in how they are manufactured, how they function in the body, and how they are administered. Because each person responds to insulin uniquely, these different treatment options allow therapy to be individualized to meet the needs of specific patients.

Varieties of insulin by species type. Insulin can be derived from several animal species. In the past, insulin derived from cows and pigs was used to treat diabetes. Synthetic human insulin, developed using recombinant DNA technology, is now used. Bovine (cow) and porcine (pig) insulins were extracted from the pancreas and processed so the human body would accept it. ${ }^{9}$ However, allergic reactions occurred in some individuals who used insulin from nonhuman sources. Human insulin is not extracted from humans but synthesized in laboratories and is identical to the naturally occurring insulin. ${ }^{36}$

Varieties of insulin by duration of action. The availability of many types of insulin with vary- ing durations of action provides options for patients with diverse needs. Insulin may be rapidacting, short-acting, intermediate-acting, or long-acting. Rapid-acting insulin (Lispro, Eli Lilly and Co., Indianapolis, IN) reaches the blood within 15 minutes after injection. It peaks 30 to 90 minutes later and may last as long as 5 hours. Short-acting (regular) insulin usually reaches the blood within 30 minutes after injection. It peaks 2 to 4 hours later and stays in the blood for about 4 to 8 hours. Intermediateacting (NPH [neutral protamine Hagedorn] and lente) insulins reach the blood 2 to 6 hours after injection. They peak 4 to 14 hours later and stay in the blood for about 14 to 20 hours. Ultralente insulin is a long-acting insulin that takes 6 to 14 hours to start working. It has no peak or a very small peak 10 to 16 hours after injection. It stays in the blood between 20 and 24 hours. Insulin glargine is a long-acting insulin with a relatively constant blood level over 24 hours without a peak.

Because it is common for people with diabetes to use more than one type of insulin, some insulins are available as combination therapies. For example, a combination of regular insulin and NPH insulin is available as a premixed solution. This can be convenient because it reduces the number of injections required to control blood glucose. However, the ratio of insulins cannot be adjusted as needed. For this reason, some patients prefer to mix their own insulin.

Varieties of insulin by method of delivery. Most often, insulin is self-injected subcutaneously 2 to 4 times per day. Traditionally, syringes are used, but special delivery devices such as insulin pumps and pens are also available. Although these devices still use a needle, some patients are more comfortable using them.

Insulin pens can be a convenient option. The pen contains a premeasured amount of insulin, but patients can use variable amounts depending on their particular needs at the time of dosing. The pen has a dial that is turned to select the amount of insulin desired. After an insulin pen has been opened, it can be stored at room temperature and reused over a period of days. 
However, patients should use a replacement needle for each injection. ${ }^{16}$

Continuous subcutaneous insulin infusion using a pump is another option for insulin delivery. Insulin pumps, which were first developed 20 years ago, provide a continuous flow of insulin to the blood that more closely mimics the way the body produces its own insulin compared to daily injections. The design and technology has evolved over the years to be more accommodating to patients. The battery-operated device is continuously worn external to the body, requiring a commitment by patients, their families, and providers to ensure its consistent, appropriate use. The use of the pump has risen sharply in the past few years, particularly among adolescents, suggesting its convenience and efficacy for maintaining good blood glucose levels without the need for injection twice to four times daily. ${ }^{37}$ Compared to traditional intensive insulin therapy, the insulin pump has achieved a $10 \%$ greater reduction in blood glucose levels when used by patients age 12 to 20 . According to results from the DCCT, this could translate to a reduction in retinopathy (diabetic eye disease) of up to $42 \% .^{38}$

Future delivery systems. Although pens and pumps have allowed people with diabetes some degree of choice and convenience, other insulin delivery devices currently in development could expand future options. The primary objective of many of these devices is to reduce or obviate the necessity of needles entirely. Inhalers containing insulin powders and liquids are now being tested, as are transdermal patch systems and oral sprays. ${ }^{15}$ Although these methods require further testing to elucidate their safety and efficacy, there is a real possibility of a noninjectable insulin in the relatively near future. Such innovations will give people with diabetes greater latitude in their choice of treatment options, allowing them to match their treatment to their own wants and needs. Inhalers and patches may work well for people who travel often, who cannot easily interrupt work or other activities to inject, or who are simply uncomfortable with needles. But each method may not work for everyone (e.g., patients with lung impairment may not benefit from inhaled insulin), nor will any one method completely remove the need for injections for everyone (e.g., inhalers will probably be adjunctive therapy to long-acting and intermediate-acting injections). The availability of alternative delivery devices and dosage forms and routes of administration will allow for greater individualization of care, perhaps encouraging greater adherence and tighter blood glucose control, and thus improving clinical and economic outcomes.

Despite many innovations, managing diabetes consistently over time is still a challenge. No one particular management strategy will work for everyone with diabetes for several reasons: the dual nature of the disease (type 1 vs. type 2), its multifactorial etiology including genetic, physiologic and environmental factors, and the diversity of individual sufferers. Therefore, identifying an effective strategy for managing diabetes is best accomplished on a case-by-case, patientby-patient basis. ${ }^{9}$ Table 3 compares diabetes management strategies in terms of indications, benefits, and major side effects.

\section{INDIVIDUALIZING CARE FOR PATIENTS WITH DIABETES}

Why is individualized pharmaceutical care needed?

Controlling diabetes demands individualized patient care for several mutually dependent reasons. First, the origin of disease in diabetes is complicated, dynamic, and multifactorial. Second, the diabetic population is becoming more diverse in age, ethnicity, overall health, and other characteristics. Third, diabetes often occurs concurrently with other medical conditions, especially in certain groups such as the elderly. These factors suggest that pharmaceutical care should be tailored to meet patient-specific characteristics and needs. Fortunately, there are many diabetes medications available in different dosage forms and quantities with varying duration of action, mechanism of action, and side-effect profiles. Despite the array of choices, there are still sufferers who 


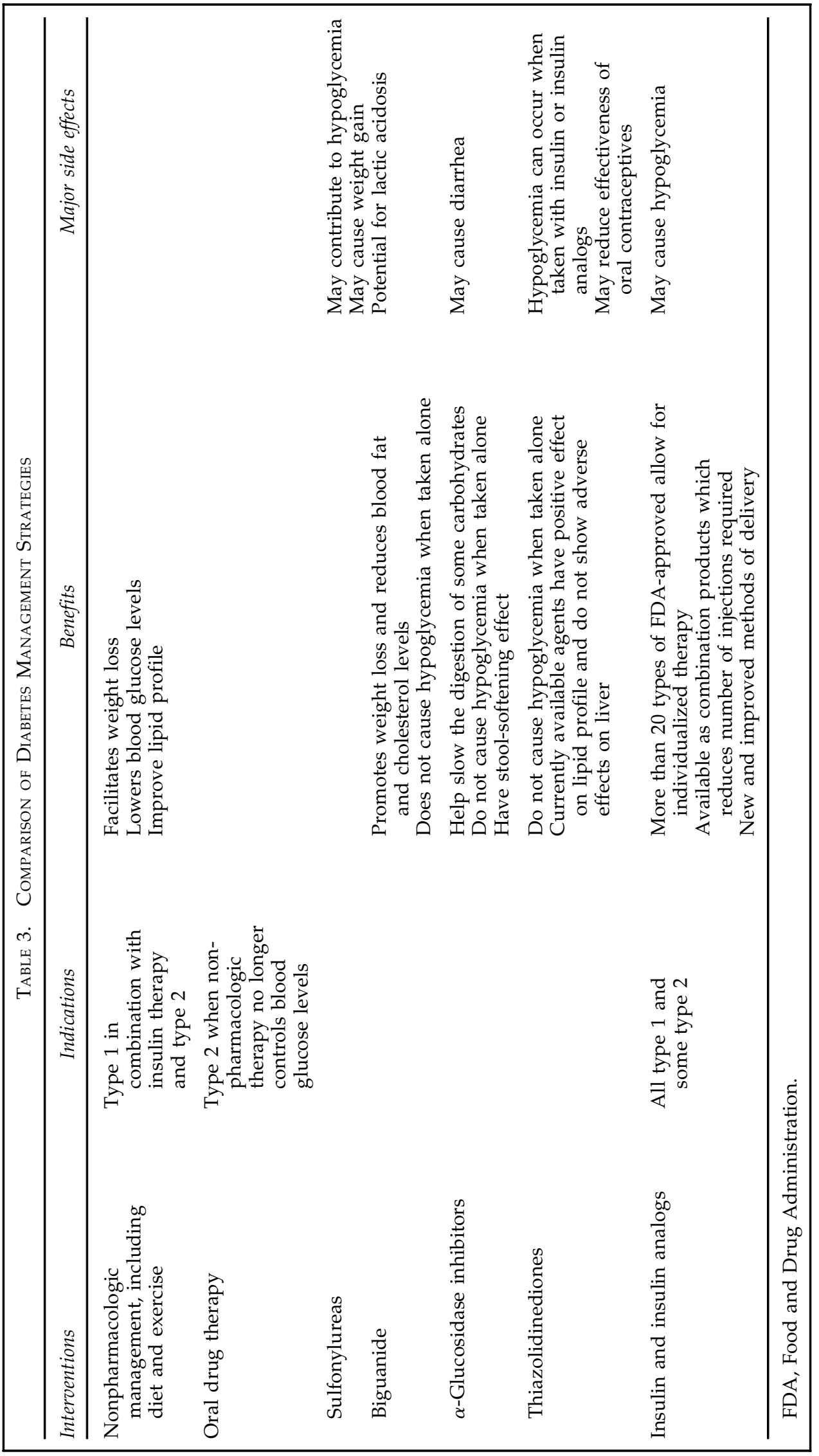


have not responded to treatment; new medications are currently in development for this therapy resistant population, and continued research efforts are warranted.

\section{A disease with a complex etiology}

Identifying and managing diabetes is complex because of its dual nature (type 1 vs. type 2) and its multifactorial origins. Genetic and environmental factors are believed to play a significant role in both types of diabetes. Type 1 diabetes usually begins in childhood and is caused by an abnormal autoimmune process that destroys cells in the pancreas, which makes insulin. ${ }^{23}$ This accounts for the total absence of insulin, the hallmark of type 1 diabetes, which requires daily insulin therapy to sustain the patient. While environmental factors, such as diet and exercise, can affect the management of type 1 diabetes, they do not cause the disease.

In type 2 diabetes, genetic and environmental causes are difficult to untangle. Studies have indicated that the condition aggregates in families, especially those with a preponderance of obese and / or sedentary family members. ${ }^{6}$ It remains unverified whether this link is genetically or environmentally determined, but in all likelihood familial type 2 diabetes probably stems from both factors. In addition, children of women who develop diabetes during pregnancy have a greater chance of developing it themselves compared to those whose mothers develop diabetes after pregnancy. This suggests prenatal involvement. ${ }^{6}$

One theory, the "thrifty genotype hypothesis," suggests that nutritional deprivation during fetal development increases the likelihood of type 2 diabetes. Some infants may have a specific genetic makeup that allows them to survive despite low levels of prenatal nutrition, but this same genotype increases their risk of developing diabetes. Metabolism and insulin resistance may also be genetically linked to diabetes, but according to diabetes researcher Peter Bennett, "genetic determinants have been difficult to elucidate because there are multiple biochemical and metabolic abnormalities, probably polygenic, and the situation is complicated by environmental factors and late age of onset." 6

In addition, physiologic changes that occur with age factor into the diabetes equation, both in terms of onset and treatment. Changes such as alterations in metabolism and body composition affect glucose tolerance in almost everyone. As people age, some impairment in glucose blood levels occurs, with average increases of 5 to $10 \mathrm{mg} / \mathrm{dL}$ per decade after age $50 .{ }^{39}$ But for more than half the population over age 65 , these age-related physiologic changes, coupled with other factors such as nutrition and activity level, lead to clinically diagnosable type 2 diabetes. ${ }^{39}$

\section{Diabetes in special populations}

All the factors implicit in the origins of diabetes-family genetics, environment and behavior, and the physiology of aging and comorbidity-contribute to subpopulations at special risk for developing the disease. Diabetes may also manifest or progress differently in these high-risk groups. Likewise, the management of diabetes in subpopulations can be varied to meet the diversity of group and individual healthcare needs. This section takes a closer look at several groups for whom diabetes prevention and individualized treatment is essential to ensuring positive health outcomes.

Diabetes in women. Diabetes affects more than $8 \%$ of all women in the United States, a percentage that is similar to that in the male population. But although men and women are at equal risk for developing diabetes, the repercussions of the disease can be particularly dangerous for women. For example, women with diabetes have an increased risk of complications during pregnancy. Also, women have a much greater chance (up to $50 \%$ higher) than their male counterparts of developing diabetic ketoacidosis, which poses a serious health threat. ${ }^{40}$

Compared to women without diabetes, women with diabetes are nearly eight times more likely to suffer from peripheral vascular disease (PVD) in which blood and oxygen flow to the lower extremities is reduced. PVD causes 
intermittent claudication (leg pain during exercise), and this condition predisposes the sufferer to a 3 to 4 times greater risk of several cardiovascular diseases. ${ }^{13,40}$

Women with diabetes who are also pregnant face the possibility of additional complications. Because the body demands more insulin during pregnancy, extremely strict blood glucose control is essential for women who are pregnant or trying to conceive. The early infant mortality rate for babies born to mothers with diabetes is as much as two to three times higher compared to children of women who do not have diabetes. Moreover, this risk increases when preconception care of the mother is inadequate or absent. Pregnant women with diabetes are at increased risk of having high birthweight newborns, thus increasing the need for cesarean section to three to four times that of the general population. Dangers also include greater risks of toxemia and hydramnios (excessive accumulation of the amniotic fluid). Both are serious health concerns for the mother and child. ${ }^{40}$

Even women who do not have diabetes when they conceive are still at risk for an alternate form of the condition known as gestational diabetes. Gestational diabetes develops in $2 \%$ to $5 \%$ of all expectant women without diabetes but resolves after pregnancy. However, gestational diabetes increases the chances of complications during pregnancy and increases the chances of both child and mother developing type 2 diabetes postdelivery. ${ }^{41}$

Diabetes in children and adolescents. Diabetes is the most common severe chronic disease of childhood. The majority of children and adolescents with diabetes are diagnosed with type 1 disease. Type 1 diabetes in children usually occurs during puberty, and the disease runs in families, suggesting a genetic component. Unlike type 2 diabetes, the incidence of type 1 diabetes is greater in whites than in other racial groups.

The number of youths with type 2 diabetes is growing. Children with type 2 diabetes tend to be overweight and slightly older at diagnosis, compared to youths with type 1 diabetes. They may have a family history of type 2 dia- betes and are also likely to be African American, Native American, or Latino.

Treatment of children with diabetes requires special consideration. Studies suggest that achieving and maintaining an optimal blood glucose level is especially important in this age group. The Pittsburgh Epidemiology of Diabetes Complications Study revealed a relationship between glycemic control and disease complications in childhood onset diabetes. ${ }^{41} \mathrm{In}$ cases where there was very poor gylcemic control, retinal complications have occurred before puberty. While the evidence is conflicting, the number of years a child lives with diabetes before puberty may correlate with an increased risk for future vascular complications. ${ }^{42}$

The nature of childhood itself uniquely affects diabetes and its management. Special considerations must be taken into account for this population. Intense activity during play or sports, high sugar and fat content of snack foods marketed toward children, and the general level of personal responsibility for self-care all significantly impact blood glucose levels and diabetes management in children. One key to successful treatment is consistent blood glucose monitoring followed by therapeutic and behavioral adjustments to reach optimal levels. ${ }^{42}$ Depending on age, children may or may not be old enough or responsible enough to understand and adhere to a program of monitoring and management. Adherence among children can be increased by intensive diabetes education for the patient and his or her family. For children taking insulin who require more flexibility in daily activity and dietary habits, four injections per day, compared to two, may result in better glycemic control. ${ }^{42}$

For all of these reasons, childhood diabetes requires early detection and individualized treatment to optimize outcomes at onset and throughout later life. More research is required to determine the special characteristics unique to children with diabetes to maximize the success of current therapies or suggest innovations that could improve diabetes care for children. Whatever therapy is selected, tight glycemic control is essential for good outcomes. 
Diabetes in the elderly. The elderly are discussed in the diabetes medical literature more than any other patient population identified in this paper. Perhaps the abundance of literature reflects the significant impact diabetes has on seniors: half of all people with diabetes are older than 55 years of age. ${ }^{40}$ Because aging is an independent risk factor for type 2 diabetes, everyone has a vested interest in understanding diabetes prevention, risk factors, and early detection. For those people who do acquire diabetes during their adult or later adult lives, understanding diabetes management options is critical as well.

What are the special considerations for older patients with diabetes? Primarily, the complications of diabetes are more prevalent and more dangerous for older individuals. Because aging reduces the body's physiologic reserves, alters its metabolism, and diminishes organ function, diabetes complications are more common in this age group. For example, the prevalence of diabetic heart disease increases with age. Likewise, kidney infections are twice as common in the 65-plus diabetic population as in the population without diabetes. ${ }^{40}$ In addition to complications caused by diabetes, the increased prevalence of comorbidities in older patients is a special concern for people with diabetes. Comorbidity, or the coexistence of two or more diseases, occurs more often with age, and its presence increases morbidity, mortality, and medical resource use. ${ }^{43}$ The specific concerns associated with diabetes with comorbidity are discussed later in this paper.

One specific concern about diabetes in the elderly is their increased risk of hypoglycemia. Because the risk of hypoglycemic episodes is greater in the aged, monitoring blood glucose levels carefully is essential, especially when hypoglycemic medications are used. First-line treatment should include diet and exercise modifications, if possible. Then, if pharmacotherapy is required to control blood glucose levels, selection should be based on the patient's individual needs.

However, some diabetes medications should generally be avoided in the elderly. For example, chlorpropamide, an oral sulfonylurea, has a long half-life and high incidence of hypoglycemia and is not preferable for most older patients. ${ }^{9}$ A better option in this class of drugs are shorter-acting, less hypoglycemic agents such as glipizide. When initiating drug therapy, elderly who may have altered metabolism should begin with half the recommended dosage given to younger patients and titrate to a therapeutic level. Also, patients with renal or hepatic insufficiency should not be treated with sulfonylureas.

Diabetes in certain racial and ethic groups: Hispanic Americans, Native Americans, African Americans face greater risks. Similar to the elderly, certain ethnic and racial groups face greater risks of type 2 diabetes and its complications. The comparative prevalence of type 2 diabetes in these groups is astounding: $24 \%$ of all Mexican Americans, 26\% of all Puerto Ricans, 11\% of African Americans, 12\% of Native Americans, and $16 \%$ of Cuban Americans have diabetes compared to $6 \%$ in the general population. Asian Americans and Pacific Islanders appear to be at greater risk also, although prevalence data are limited. ${ }^{2}$ In one specific tribe of Native Americans residing in Arizona, the prevalence of type 2 diabetes is higher than in any other group worldwide, with $50 \%$ of adults ages 30 to 64 having the disease. Among Native Americans overall, complications from diabetes are the most frequent cause of death from disease.

Several factors account for higher rates of diabetes morbidity and mortality in ethnic groups. Environmental factors may include access to quality, affordable healthcare, varying levels of functional health literacy, effective patient education, diet, exercise, and other health behaviors including alcohol and tobacco use. Although these socioeconomic and behavioral factors play a considerable role, recent research concludes that ethnicity alone is strongly associated with diabetes and its control in some minorities. ${ }^{44}$ This finding implicates genetic variables as risk factors for type 2 diabetes; if so, recent and ongoing research in genomics and biotechnology could offer improved care options for subpopulations with a predisposition 
to the disease. At any rate, the conclusions suggest that continued efforts to support biotechnological innovation are warranted to promote equitable diabetes care for minority groups.

\section{Diabetes and comorbidity}

Comorbid conditions are common in patients with diabetes. In many cases, diabetic complications account for these conditions; in others, the coexisting condition(s) precedes or occurs independent of diabetes. In either situation, comorbidity requires specialized care regimens to optimize clinical outcomes. Comorbidity often generates disease synergies more debilitating than the additive effects of each individual disease. Common comorbidities in patients with diabetes include obesity, cardiovascular diseases, depression, and nerve, eye, and kidney diseases. While a discussion of all diseases that commonly coexist with diabetes is too broad for the scope of this paper, the following sections focus on two major comorbid illnesses associated with diabetes: cardiovascular conditions and depression.

Diabetes and cardiovascular conditions. Researchers have extensively studied the connection between diabetes and cardiovascular disease (CVD). In the United States, cardiovascular disease is the leading cause of death in both men and women with diabetes. The mortality rate associated with CVD is significantly higher in women than in men and increases with advancing age in both men and women. ${ }^{13}$

When treating patients with comorbid diabetes and CVD, minimizing the deleterious effects of both conditions is essential. If the treatment of one disease is emphasized over the other, the undertreated disease can exacerbate the seemingly well-managed one. However, intensive risk factor modification improves the prognosis of patients with comorbid diabetes and CVD. ${ }^{45}$ Many risk factors, such as poorly controlled blood glucose levels, hypertension, and dyslipidemia, can be minimized with drug therapy.

The Multiple Risk Factor Intervention Trial
(MRFIT), a 12-year analysis of coronary heart disease (CHD), revealed higher mortality rates for patients with diabetes. Hypertension, hypercholesterolemia, and cigarette use further increased these rates in individuals with diabetes. ${ }^{45}$ Controlling these risk factors with pharmacotherapy can reduce the risk of cardiovascular events. Strict control of blood pressure and blood glucose levels appears to be a primary therapeutic goal. ${ }^{13}$ Blood pressure can be reduced by using various classes of antihypertensive agents such as diuretics, angiotensin-converting enzyme (ACE) inhibitors, $\beta$ blockers, and calcium channel blockers. Within each drug class, there are numerous agents from which to choose. The specific choice of an antihypertensive agent is important in that, first and foremost, it must reduce blood pressure, and second, it should be selected to meet specific patient needs, taking into consideration age, comorbidities, and other health factors.

Antihypertensives agents that increase blood glucose levels (e.g., diuretics) should be avoided in patients with diabetes. Recently, aspirin therapy has also been recommended by the American Diabetes Association as a consideration for adults with diabetes who have CVD or CVD risk factors. While nearly every diabetic adult in the United States has at least one risk factor for CVD, only $20 \%$ take aspirin regularly, 46 suggesting that undertreatment may play a large role in inadequately controlled risk factors for comorbid diabetes and CVD.

Diabetes and depression. Although not usually indicated as a complication of diabetes in the same way as heart disease and obesity, depression is a common comorbid condition facing many patients. A recent study by Anderson and colleagues $^{47}$ concludes that having diabetes doubles the odds of comorbid depression. This National Institutes of Health (NIH)-sponsored meta-analysis of 42 diabetes/depression studies showed that major depression was present in $14.7 \%$ and elevated depression symptoms in $26 \%$ of diabetic patients. ${ }^{47}$

Now that the medical community generally acknowledges that mental status can impact the 
course of comorbid physical illnesses, ${ }^{11}$ these rates of depression are a serious consideration in terms of treating people with diabetes. However, studies have suggested that even in the general population, depression is undertreated..$^{48}$

Inadequate treatment of depression in patients with diabetes further compromises health status for several reasons. First, a depressed patient may not be motivated to follow the rigid self-care routines required to maintain good blood glucose control. Second, depression often results in appetite and weight fluctuations that can impact diabetes progression or encourage undesirable and dangerous hypoglycemic or hyperglycemic events. In addition, diabetes can negatively impact major depression by increasing the frequency of recurrent depressive episodes in the five years following diagnosis. ${ }^{11}$ Diabetes increases the risk of subclinical depressive symptoms as well. ${ }^{47}$

Recent reports reveal that treating comorbid depression in patients with diabetes can improve glycemic control without interfering with diabetes medications. Both tricyclic antidepressants and selective serotonin reuptake inhibitors (SSRI) have shown efficacy in treating depression. ${ }^{49}$ However, SSRIs offer fewer side effects and a lower risk of a direct hyperglycemic effect. ${ }^{11,50}$ A more recent study of patients with diabetes with depression showed that after only 8 weeks of treatment with the SSRI fluoxetine, depressive symptoms decreased, and glycemic control showed improvement. ${ }^{51}$

\section{PROMOTING QUALITY, COST- EFFECTIVE DIABETES MANAGEMENT THROUGH INDIVIDUALIZED CARE}

With the efficacy of intensive, individualized diabetes treatment established, the medical research community is now focused on how to achieve this outcome in a cost-effective manner. The primary cost of treating diabetes comes from short-term hyperglycemia and long-term complications. ${ }^{52}$ One analysis of intensive treatment of type 2 diabetes found that achieving normal blood sugar levels would re- duce the incidence of blindness by $72 \%$ and end-stage renal disease by $87 \%$, lower extremity amputation by $67 \%$, and raise life expectancy by 1.4 years. ${ }^{53}$ Importantly, these facts and figures imply that good clinical and economic outcomes are aligned. By reducing the incidence of diabetes complications, costs would decrease as well.

Improved clinical practice in diabetes management is one step toward quality, cost-effective outcomes. In July 1995, the American Diabetes Association convened a panel of experts to develop a consensus statement on the pharmacologic treatment of hyperglycemia in type 2 diabetes. The panel, relying on evidence from the DCCT, recommended that if progress toward glycemic goals was not apparent within a 3-month period after initiation of diet and exercise, then use of a pharmacologic agent is appropriate. ${ }^{26}$ Before the availability of antidiabetic drugs, approximately $35 \%$ of type 2 patients were treated with insulin alone. While clinically effective for glycemic control, insulin therapy requires diligent compliance on the part of the patient. For some individuals-especially children, the elderly, and patients with low functional health literacy-complying with a rigid drug regimen is a considerable task. Thus, achieving this compliance presents the clinician with the challenge of educating the patient about self-administration and dose adjustment. ${ }^{54}$ Moreover, compliance is linked to factors such as side effects, ease of administration, affordability of medication, and the patient's perception of effectiveness. Therefore, selecting the specific combination of medications that will meet an individual patient's needs is essential to encouraging compliance and improving outcomes.

Fortunately, the array of independently acting antidiabetic medications available makes possible a number of combination therapies, and recent research focusing on the efficacy of particular combinations has revealed opportunities where judicious selection among antidiabetic agents of similar efficacy may have beneficial effects on complications of diabetes or comorbid conditions. 
The availability of antidiabetics agents with complementary mechanisms of actions allows clinicians to select treatments that have additive benefits and avoid detrimental side effects.

A meta-analysis of nine randomized controlled trials published since 1957 was conducted in 1999 to compare the efficacy of biguanides and sulfonylureas. Although the efficacy is similar for biguanides and sulfonylureas, sulfonylurea treatment was associated with an increase in body weight, and biguanides with a decrease, suggesting that a biguanide may be preferred for obese patients. ${ }^{55}$

\section{DIABETES PUBLIC POLICY IMPLICATIONS}

Healthy People 2010, the nation's comprehensive plan for improving public health, includes goals for reducing the burden of diabetes and improving the quality of life for all persons who have or are at risk for the disease. Specifically, it states a goal of reducing by $10 \%$ the number of deaths from CVD in persons with diabetes. Over the past ten years, diabetes has been a leading cause of death in the United States, primarily due to diabetes-related CVD. Achieving reductions in addressable risk factors, such as uncontrolled hypertension, smoking, and high cholesterol, could significantly reduce the incidence of CVD in persons with diabetes.

Related goals in Healthy People 2010 are directed toward reducing the incidence of other costly, life-shortening complications among patients with diabetes, including end-stage renal disease, blindness, lower extremity amputation, and complications of pregnancy. Because weight reduction ameliorates metabolic abnormalities characteristic of diabetes and may help prevent complications of the disease, addressing overweight and obesity is of major importance in reaching this goal.

To reach the Healthy People 2010 goals, there must be improvements in the practice of healthcare for people with diabetes. Measurement and monitoring of glycosylated hemoglobin $\left(\mathrm{HbA}_{1 \mathrm{c}}\right)$ levels is the gold standard for glycemic control as specified in guidelines, yet it is still underused, especially in primary care. ${ }^{56}$ There is considerable interest in the effect of managed care on diabetes treatment patterns. Interest has grown with the spread of managed care coverage to higher-risk Medicare beneficiaries in the late 1990s. Referral to out-of-network physicians and specialists is a more complex process in managed care; therefore, diabetics are relying increasingly on care and education from their primary care physicians.

The long-term impact of managed care on diabetes treatment patterns is still not known; however, some believe increased use of oral antidiabetic agents may be attributed in part to managed care practices. Moreover, with the introduction of diabetes treatment-related performance measures into the National Committee for Quality Assurance, Health Plan Employer Data and Information Set (HEDIS ${ }^{\circledR}$ ), many managed care plans have developed comprehensive diabetes treatment programs to promote early detection of diabetes and related conditions through educational programs and screenings. While front-end financial investments such as increased drug spending and diabetes disease management are costly, the potential return-on-investment could be doubly advantageous. By improving clinical outcomes through better diabetes prevention and management, overall expenditures, especially those associated with long-term complications, could be significantly reduced.

\section{DIABETES: a tool for a complex condition}

Creating cost-effective diabetes management plans to meet the individual needs of a diverse patient population is dependent on many factors. When one or more of these factors are overlooked, therapy plans may be less than optimal, clinical outcomes less positive than expected, and financial returns inconsequential. A complex condition such as diabetes requires a comprehensive strategy to address clinical and economic outcomes adequately. To help ensure that all factors are considered, we have designed a mnemonic tool to remind patients, providers, and policy-makers of the many facets of indi- 
vidualized diabetes care. Our DIABETES device addresses what we believe to be the essential components of individualizing pharmaceutical therapy in the treatment of diabetes.

Delivery: Matching a dose delivery method with a patient and his or her special needs is a key step toward ensuring compliance and encouraging good glycemic control. Patients who are comfortable with their insulin delivery system will be more willing and better able to adhere to their diabetes plans.

Integration of Care: When all care providers and sites are well integrated, the opportunity to maximize the quality of diabetes care is at its peak. Integrated care includes communication among physicians, specialists, pharmacists, nurses, and other people involved in the patient's care process. It also means that systems and programs have been implemented to easily facilitate such communication. For example, information-sharing systems supported by Web-based and electronic tools can connect all of a patient's care providers, and in some cases even the patient, to form a network of integrated care for each individual. Also, integrated disease management (DM) programs that address multiple therapeutic areas, such as the combined diabetes and heart disease DM programs that have recently evolved, are creating care processes that address the complexity of diabetes and comorbid disease.

Adherence: Adherence may be more important in diabetes than in any other disease state. Adherence promotes strict glycemic control, and glycemic control is the best method for controlling diabetes and preventing its complications. That is why promoting adherence is vital to diabetes care. Adherence can be encouraged through offering intensive diabetes education, creating convenient dosing regimens to fit individual lifestyles, addressing the emotional and psychological needs of patients with diabetes, minimizing medication side effects, providing following-up care through $\mathrm{PCP}$, nurse practitioners or structured DM programs, and designing accessible and affordable diabetes management plans for individual patients.
Behavior Change: To promote individualized care for people with diabetes, behavior change will need to occur at the patient, provider, and policy level. We know this because although effective diabetes diagnostics, therapies and treatment guidelines exist, there are still nearly 5 million cases of undiagnosed diabetes in America. Of the patients who are diagnosed, many still suffer uncontrolled blood glucose levels that can lead to costly clinical complications. The tools to prevent and manage diabetes are out there, and new and improved tools are developed every day. But the systems, processes, and individual behaviors of all diabetes stakeholders must maximize the use of these tools if the fight against diabetes is to succeed. Behavior change can be affected through education, incentives, policies, and regulations. All of these methods must be explored to enhance diabetes outcomes.

Education: Education that occurs at every contact point between the patient and care providers is needed. Providers who take advantage of every "teachable moment" will be most successful in helping their patients develop the skills and attitudes that they need to manage their diabetes. Opportunities for educating patients may be less formal, such as impromptu discussions during office or pharmacy visits, or more structured, such as planned patient education meetings offered as part of a larger diabetes DM program. Education can be provided in many modalities-patient literature, videos, one-on-one, or group instruction. Studies have shown clearly that a patient's functional health literacy is directly connected to the kind of care he or she seeks and receives and to health outcomes. Therefore, maximum effort is needed to provide diabetes education that reaches all patients.

Treatment Options: Each year, the American Diabetes Association, with the support of expert clinicians and researchers, reviews and revises treatment guidelines and recommendations for caring for people with diabetes. These guidelines, along with the myriad of pharmacologic and nonpharmacologic treatment option for diabetes, provide the foundation for 
quality diabetes care. Yet the existence of guidelines and treatment options is not enough. The wide range of diabetes medications must be made available for all patients if individualized care is to be achieved.

Economics: Healthcare coverage, be it private or publicly funded, that limits access to the full span of diabetes medications could compromise health outcomes for patients. Moreover, we know that compromised health outcomes in diabetes lead to extraordinarily expensive complications. The front-end costs associated with quality preventive diabetes care and management are an investment that has the potential to alleviate at least a portion of the financial burden associated with the high costs of uncontrolled diabetes. Controlling diabetes may be expensive, but is it as expensive as allowing the disease to run rampant through our national and personal healthcare resources?

Special Circumstances: For pharmaceutical care to be truly individualized, any special circumstances regarding the patient need to be considered when developing a treatment plan. Although this is to some degree true for any disease or medical condition, it is especially relevant in the case of diabetes because of its pervasiveness. Diabetes infiltrates all aspects of an individual's life, from what to choose for breakfast to how to schedule daily activities around administering medications and monitoring blood glucose levels. Therefore, any situations that are unique to an individual patient have the potential to impact the diabetes care plan. Examples of such factors include socioeconomic issues like living environment, transportation, and financial resources as well as clinical issues such as comorbidities and general health status. At least one individual on the patient's diabetes care team needs to be prepared to discuss any issue that might impact the diabetes treatment plan.

\section{REFERENCES}

1. Centers for Disease Control and Prevention. Diabetes: A serious public health problem. At A Glance. 2001; $1-6$.
2. Diabetes. Washington, DC: The National Hispanic Council on Aging; 1999:1-7.

3. Diabetes in Hispanic Americans. Bethesda, MD: NIDDK National Diabetes Information Clearinghouse; 2001:1-15.

4. Diabetes in African Americans. Bethesda, MD: NIDDK National Diabetes Information Clearinghouse; 2001: $1-12$.

5. Diabetes and its impact on the elderly. MultiMedia Health Care/Freedom LLC, ed. Clin Geriatr 2000;1-2.

6. Bloomgarden ZT. American Diabetes Association annual meeting 1996: The etiology of type II diabetes, obesity, and the treatment of type II diabetes. Diabetes Care 1996;11:1311-1315.

7. Jenkins DJA, Jenkins AL. Nutrition principles and diabetes: A role for "lente carbohydrate"? Diabetes Care 1995;18:1491-1498.

8. White E, Danish A. The elderly as the new consumer of health care. In: Nash D, Manfredi M, Bozarth B, Howell S, eds. Connecting with the New Healthcare Consumer: Defining Your Strategy. New York: McGraw-Hill, 1999:413-432.

9. Campbell L, Hunter K. Recent advances in the treatment of diabetes mellitus: special considerations in the elderly. Clin Geriatr 2000;1-7.

10. Reference removed at press time.

11. Watkins CE. Diabetes, depression, and stress. Northern County Psychiatric Associates 2001;1-10.

12. Greenfield S, Rogers W, Mangotich M, Carney MF, Tarlov AR. Outcomes of patients with hypertension and non-insulin dependent diabetes mellitus treated by different systems and specialties. Results from the medical outcomes study. JAMA 1995;274:1436-1444.

13. Kaseta JR, Sowers JR. Diabetes mellitus and cardiovascular disease in the older woman. MultiMedia Health Care/Freedom LLC, ed. Clinical Geriatrics 2000.

14. Diabetes II: Managing Diabetes in the Elderly. Online Learning Center 2001;4-4.

15. Alderman C. Special delivery. Nurs Standard, 2000; 14:18.

16. Medicines for People with Diabetes. U.S. Department of Health and Human Services 1997;1-28.

17. Froguel P, Mulligan B. Diabetes reveals its genes. Magazine for European Research 2001;26:1-3.

18. Alliance for Aging Research. The Dawn of Gero-Technology: Pioneers in Aging and Regenerative Medicine. 2000;1-20.

19. Parexel Medical Marketing Services. Biotechnology's impact on diseases of the elderly: A white paper. Biotechnology Industry Organization 2000;1-101.

20. Center for Disease Control and Prevention. Diabetes Surveillance 1999. U.S. Department of Health and Human Services.

21. Harris I, Flegal K, Cowie C, Eberhardt M, Goldstein $\mathrm{D}$, Little $\mathrm{R}$ et al. Prevalence of diabetes, impared fasting glucose, and impaired glucose tolerance in U.S. adults. Diabetes Care 1998;21:518-524.

22. American Diabetes Association. Type 2 diabetes in children and adolescents. Diabetes Care 2000;23:381.

23. Gillespie J. Disease management: Balancing cost and 
quality. Studies on the benefits of disease management services for the treatment of diabetes. In: The Value of Disease Management. Reston, VA: National Pharmaceutical Council, 2000:1-61.

24. Food and Drug Administration. Diabetes demands a triad of treatments. FDA Consumer 1999;1-10.

25. American Diabetes Association. Economic consequences of diabetes mellitus in the U.S. in 1997. Diabetes Care 1998;21:296-309.

26. American Diabetes Association. Clinical Practice Recommendations. Diabetes Care 2001;24(Suppl 1):1-133.

27. The Diabetes Control and Complications Trial Research Group. The effect of intensive treatment of diabetes on the development and progression of longterm complications in insulin-dependent diabetes mellitus. N Engl J Med 1993;329:977-986.

28. Ramlo-Halsted B, Edelman S. The natural history of type 2 diabetes. Implications for clinical practice. Primary Care 1999;26:771-789.

29. American Diabetes Association. Implications of the United Kingdom Prospective Diabetes Study. Diabetes Care 1998;21:2180-2184.

30. Edelman S, Henry R. Diagnosis and Management of Type 2 Diabetes, 2nd ed. Berkley, CA: University of California, 1998.

31. Purnell J, Hirsch IB. New oral therapies for type 2 diabetes. American Family Physician 1997;56:18351842.

32. Lebovitz H. Glucosidase inhibitors as agents in the treatment of diabetes. Diabetes Rev 1998;6:132-145.

33. Diabetes Drug Database. CoreyNahman.com, Pharmaceutical News and Updates, 1-4.

34. Glucovance (Glyburide and Metformin Tablets) approved for type 2 diabetes. Bristol Meyers Squibb 2001;1-4.

35. American Diabetes Association. About insulin. 2001; Available at: www.diabetes.org.

36. Insulin: the drug of life for diabetics. 2001; Available at: about.org.

37. Maniatis AK, Klingensmith GJ, Slover RH, Mowry CJ, Chase HP. Continuous subcutaneous insulin infusion therapy for children and adolescents: An option for routine diabetes care. Pediatrics 2001;107:351-356.

38. Boland EA, Grey M, Oesterle A, Fredrickson L, Tamborlane WV. Continuous subcutaneous insulin infusion. A new way to lower risk of severe hypoglycemia, improve metabolic control, and enhance coping in adolescents with type 1 diabetes. Diabetes Care 1999;22:1779-1784.

39. Bourne K. Better Management of Geriatric Diabetes. 2001; Available at: www.medinfosource.com.

40. Diabetes Facts and Figures. American Diabetes Association 2000;1-27.

41. Orchard TJ, Dorman JS, Maser RE, Becker DJ, Ellis D, LaPorte RE, et al. Factors associated with avoidance of severe complications after $25 \mathrm{yr}$ of IDDM. Pittsburgh Epidemiology of Diabetes Complications Study I. Diabetes Care 1990;13:741-747.

42. Dorchy H, Roggemans MP, Willems D. Glycated hemoglobin and related factors in diabetic children and adolescents under 18 years of age: A Belgian experience. Diabetes Care 1997;20:2-6.

43. Nash D, Koenig J, Chatterton M. Why the elderly need individualized pharmaceutical care. 1st ed. Philadelphia, PA: National Pharmaceutical Council, 2001:1-18.

44. Bermudez OI, Tucker KL. Type 2 Diabetes Mellitus Among Elderly Latinos. Boston, MA: Tufts University, 2000.

45. Bloomgarden ZT. American Diabetes Association 60th Scientific Sessions, 2000: Cardiovascular Disease in Diabetes. Diabetes Care 2001;24:399-404.

46. Rolka DB, Fagot-Campagna A, Narayan KM. Aspirin use among adults with diabetes: Estimates from the Third National Health and Nutrition Examination Survey. Diabetes Care 2001;24:197-201.

47. Anderson RJ, Freedland KE, Clouse RE, Lustman PJ. The prevalence of comorbid depression in adults with diabetes: A meta-analysis. Diabetes Care 2001;24:10691078 .

48. Gelenberg AJ. Depression is still underrecognized and undertreated. Arch Intern Med 1999;159:1657-1658.

49. Gomez GE, Gomez EA. The use of antidepressants with elderly patients. J Psychosoc Nurs Mental Health Serv 1992;30:21-26.

50. Lustman PJ, Griffith LS, Clouse RE, Freedland KE, Eisen SA, Rubin EH, et al. Effects of nortriptyline on depression and glycemic control in diabetes: Results of a double-blind, placebo-controlled trial. Psychosom Med 1997;59:241-250.

51. Lustman PJ, Freedland KE, Griffith LS, Clouse RE. Fluoxetine for depression in diabetes: A randomized double-blind placebo-controlled trial. Diabetes Care 2000;23:618-623.

52. US Department of Health and Human Services. Healthy People 2010: Understanding and Improving Health. 2nd ed. Chapter 5: Diabetes. Washington, DC: Public Health Service 1991.

53. Eastman R, Javitt J, Herman W. Model of complications of NIDDM. Analysis of the health benefits and cost-effectiveness of treating NIDDM with the goal of Normoglycemia. Diabetes Care 1997;20:735-744.

54. DeFronzo R. Pharmacologic therapy for type 2 diabetes mellitus. Ann Intern Med 1999;131:281-303.

55. Johansen K. Efficacy of metformin in the treatment of NIDDM. Meta-analysis. Diabetes Care 1999;21:33-37.

56. Deichmann R, Castello R, Horswell, Friday K. Improvements in diabetic care as measured by $\mathrm{HbA}_{1 \mathrm{c}}$ after a physician education project. Diabetes Care 1999;22:1612-1616.

Address reprint requests to: David B. Nash, M.D., M.B.A. Office of Health Policy and Clinical Outcomes Thomas Jefferson University Hospital 1015 Walnut Street, Suite 115 Philadelphia, PA 19107

E-mail: David.Nash@mail.tju.edu 